\title{
Patients User Experiences with Technologies in Health Service Industry
}

\section{Sara Brickel ${ }^{1}$, Enid Montague ${ }^{2 *}$ and Woodrow W. Winchester ${ }^{3}$}

${ }^{1}$ B.S. Systems Engineering, Marine Corps System Command Quantico, USA

${ }^{2}$ Department of Industrial and Systems Engineering at the University of Wisconsin-Madison, USA

${ }^{3}$ Department of Industrial \& Systems Engineering at Virginia Polytechnic Institute and State University, USA

\begin{abstract}
The purpose of this exploratory study was to identify usability problems encountered by patients, as passive users, during the interpretation of technologies that are used during patient care by clinicians and nurses. Participants were asked to interpret key artifacts in the health information technology display and determine potential outcomes. The results from this study indicate that the majority of participants were not able to accurately interpret information from the display, which may have implications for patients' health care experiences and decision-making. Patients' perceptions of technologies used while seeking and receiving health services can be explored using human-computer interaction methods, theories, and design tools.
\end{abstract}

Keywords: Patient; User experience; Heuristic evaluation; Medical technology; Health care; Human-computer interaction

\section{Introduction}

Health care providers manage and monitor patient health statuses and information with medical information and communication technologies. During medical events, patients have user experiences with the technologies used in their care, that may affect their relationships with their care providers and their assessments of the care they receive [1]. In some health care work systems, patients' relationships with technologies have led them to request technologies that may be unnecessary or risky and reject technologies that may be needed [2-4]. This trend is particularly represented in obstetrics, where patients may request or reject the use of technologies like electronic fetal monitors. This paper explores the electronic fetal monitor as a health information and communication technology that is used by health care providers to make decisions about patients' health status, but argues that patients, as passive users $[1,5]$ also make decisions about technologies that are used in their care. In particular, healthcare technologies that do not include patients as user stakeholders in the design process may incorrectly influence patients to make poor decisions about the technologies that are used in their care. Human computer interaction (HCI) methods were used in the study to understand patients' perceptions of technologies used in their care with the overall goal of demonstrating the importance of including patients' as user stakeholders in the design of health technologies used in their care.

\section{Health Care as Service}

Health care is often classified as a complex service system, a combination or recombination of three central components: people, processes, and products [6]. The people component reflects stakeholders such as patients, clinicians, and other health care workers possessing behaviors, values, and knowledge. Processes include the means by which activities and/or tasks are conducted including collaboration, customization, etc. Products include entities such as technologies and infrastructures.

Health care provides as an output, services and products in support of improving the health and well-being of individuals and/or populations. Comparable to other services, healthcare offers unique characteristics that suggest a more holistic framing and understanding in design. Berry and Bendapudi [7] assert that within healthcare
- Customers (e.g. patients) are sometime sick.

- Customers are often reluctant.

- Customers relinquish privacy.

- Customers need "whole person" service.

- Customers are at risk.

- Clinicians are stressed.

These contextual factors could impact the service delivery experience. Consideration of these factors in design, calls for the use of methods and approaches that support a more integrated view or framing of the system.

Moreover, health care can also be characterized as a sociotechnical system comprised of several subsystems [8]. Subsystems that comprise the overall health care system include the technological subsystem, personal subsystem, external environment, internal environment and the organization. Failures to consider health care's sociotechnical characteristics in design efforts could offer suboptimal results. This is of particular importance in the design of health technologies.

The prevalence of technology in health care is growing. From record keeping to surgical support to patient monitoring, the "reach" and "touch-points" of health technologies are growing. In designing these technologies, Coiera [9] asserts that designs should not be restricted to technological systems alone but broadened in scope through an understanding of their relationships to social structures, the impacted stakeholders (e.g. clinicians and patients) and their relationships to one another, and associated implications to the service delivery experience.

*Corresponding author: Enid Montague, Ph.D, 3017 Mechanical Engineering, Industrial and Systems Engineering, University of Wisconsin-Madison, Madison, WI 53706, USA, Tel: 608890 3546; E-mail: emontague@wisc.edu

Received February 08, 2012; Accepted February 28, 2012; Published March 06, 2012

Citation: Brickel S, Montague E, Winchester WW (2012) Patients User Experiences with Technologies in Health Service Industry. J Health Med Informat S7:002. doi:10.4172/2157-7420.S7-002

Copyright: (C 2012 Brickel S, et al. This is an open-access article distributed under the terms of the Creative Commons Attribution License, which permits unrestricted use, distribution, and reproduction in any medium, provided the original author and source are credited. 
The case for a more patient-centric approach to the design of health technologies has been made, with regards to technologies that patients use directly [10]. The importance of lay person's understanding and trust in medical technologies has also made. Researchers have explored lay people's trust in their abilities to use publicly available lifesaving technologies such as automatic external defibrillators and found that lay person's perceptions of trust are important to how and when technologies are used [11]. The notion of a passive user of a technology has been explored in previous research in obstetrics [1,12,]. Inbar and Tractinsky [5] describe passive user interactions as "incidental users". As detailed by Inbar and Tractinsky [5], the incidental user:

"While not 'users' in the traditional sense, incidental users are affected to various degrees by the system and by those who directly interact with it. They have considerable interest in the information presented by the system, usually as the recipients of a service. Incidental users may also be an important source of information for the system, thus taking the role of 'co-user'.

The implications to design for passive users are rich and are exemplified in the studied context - fetal heart monitoring. In this context, while the information being provided by the monitor is primarily geared towards the health care workers, because of the context and proximity \& visibility of the display to the patient, during the course of the interaction, is seeking also clues or cues for any sign - meaningful information - that might help them understand what is happening. Coins these cues "signifiers" and asserts that these cues should be provided by the technology.

In providing these cues, the challenge is to design not just a "technology" but, an "experience" through a more holistic perspective of viewing the patient as a user. Bate et al. [13] advocate for "mechanisms for creating a patient experience" that at the very least does "not induce fear, anxiety, confusion, uncertainty or panic in the patient" and "at best is smooth, natural, and trouble free \& looks and feels good." The domain of obstetrics offers a clear opportunity for exploring this notion of designing for the passive or incidental user.

\section{Obstetrics as a Health Service Domain}

Obstetrics is the practice that is concerned with the medical care of women during pregnancy, childbirth and postpartum. Ninety nine percent of all births in the United States are delivered in a hospital, unlike other medical procedures such as heart surgery or an appendectomy most people will have an experience with the obstetric work system, most women will give birth in a hospital and most people will be born in a hospital [14]. In alignment with the high number of people who experience the obstetric system, one of the more common surgical procedures in the United States is a cesarean section. Concurrently, the United States health care system is experiencing an obstetric crisis; malpractice rates of suit have increased so rapidly that many physicians have had to discontinue practice. Automated technologies, such as electronic fetal monitors allow providers to monitor several births remotely, thus enhancing obstetrics systems abilities to monitor more births with fewer providers. Coincidentally, patients' decision making about malpractice is often related to experiences with electronic fetal monitors as it creates a record of the birth process and associated clinician decision-making. Studies have also found that clinician concerns about malpractice are related to an increase in cesarean section deliveries [15]. Cesarean sections are more risky for the woman giving birth and more costly to the health care system. Obstetric systems are different from other health care sub systems because of the types and roles of patients, the care providing process, the measurements during the process, the technology used and possibly most importantly the pervasiveness of media representations of the process.

The electronic fetal monitor tracks the heartbeat of the baby and the contractions of the mother during labor [16]. When a woman in labor has a contraction, blood supply to the placenta is temporarily cut off causing the baby to rely on the oxygen previously available in the placenta [17]. If there is not enough oxygen available, there is a possibility of complications, indicated by a change in the baby's heart rate [18]. In external electronic fetal monitoring, two straps are secured around the mother's abdomen; one has a pressure gauge that can track uterus changes indicating contractions, called a tocodynamometer or toco, and the other has an ultrasound device used to measure the heartbeat of the fetus, called an ultrasonic transducer [19] (see Figure 1). In telemetry monitoring, the mother is able to be mobile and feedback from the monitor is transmitted through a radio frequency transmitter [20]. In internal electronic fetal monitoring, an electronic probe is attached to the fetus's scalp, internally producing an electrocardiogram [18].

Data from the baby's heartbeat and the mother's contractions are transmitted and displayed on a monitor and printed out as a graph on paper, where physicians and mothers can view the progress of the baby [18]. Health care providers use this information, to determine if the baby is in distress and to take medical action if necessary. Evaluating whether or not a baby is in distress consists of examining the contraction activity, the baby's heart rate, and the correlation between the two. As it is common to be connected the fetal monitor throughout the duration of labor, the mother, as well as family and friends in the delivery room, often watch the monitor throughout labor to try to understand the status of the fetus as well. This behavior is expressed in a quote from an expecting father reflecting on his experiences with fetal monitoring:

"My wife looked like she was tied down, and no matter what I did, the machine kept drawing me to it. I just couldn't keep from watching the beeps..."-Kevin ${ }^{1}$

Given that the intended consumer of the output of the fetal ${ }^{1}$ Quote 1: http://www.childbirth.org/articles/efmfaq.html\#personal

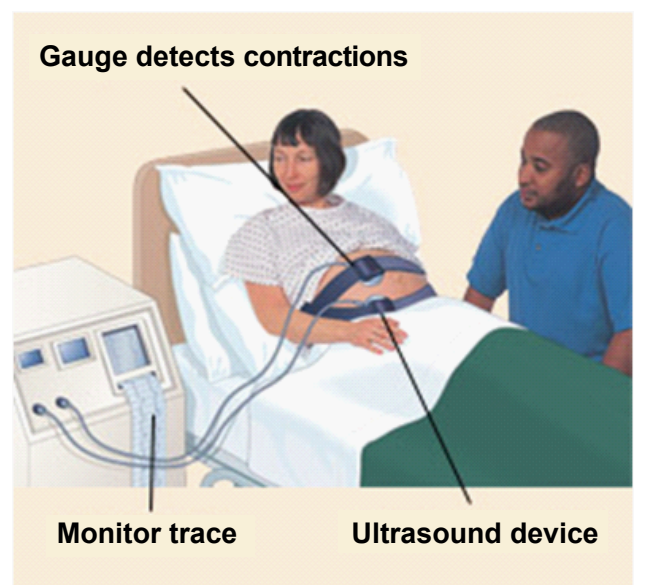

Figure 1: Electronic fetal monitor used during birth with patients. 
monitor is the health care provider, patients and family members may misinterpret the fetal monitor display output and believe the baby is in distress, which results in unnecessary \& additional patient stress and medical practitioner distraction [19]. An expecting mother echoes this sentiment in recalling her experiences with fetal monitoring:

"The EFM becomes the center of attention in the room. My labor was "risky" and although we did not have continuous fetal monitoring, we did have periodic monitoring. While the monitor was on, all eyes and ears were glued to the machine, and each dip in my baby's hear rate caused me to worry. Unnecessarily worry. The baby's heart rate is supposed to be variable" -Chris ${ }^{2}$

Clearly, from all stakeholders, both direct and passive perspectives, a proper interpretation of the fetal monitor's output is crucial to the service experience and suggests the need for usable device designs. Usability in medical devices, in general, is garnering much attention as technology use in the care continuum has grown. Design standards do exist, for example, IEC 62366:2007 specifies a process for incorporating usability within the design and development lifecycle of medical devices.

Specific to the electronic fetal monitor, much work is happening in consumer health technology design and implementation. Portable designs have now entered the consumer marketplace that allows the expectant parents to monitor and/or listen to the heart rate of the fetus for non-clinical purposes. This movement, the use of home-based monitoring technologies, coupled with more "informed" parents has increased the awareness of usability by device designers in more pervasive clinical-based designs. Philips, for example, has marketed its new line of electronic fetal monitors, the Avalon FM20 Antepartum and Avalon FM30 Intrapartum, as "user friendly in every way" [21]. This line is touted as featuring an "ergonomic design (that) projects a high-touch image to expectant parents with high-tech performance for the care team". These efforts by Philips suggest that device designers are realizing the impact of the evolving role of fetal heart monitoring technologies in the delivery process and are offering accommodating solutions.

In this study, we explored human computer interaction issues associated with electronic fetal monitors from the perspective of laypersons without any medical training, such as patients and family members; reflective of passive users of the studied technology. Studies of laypersons interactions with health technologies have been conducted [11], but research on these types of users is still underexplored. We use a participatory approach to understand technologies used in health care services of which passive users are of consideration. Specifically, we used widely methods in human-computer interaction such case scenarios, usability heuristics and cognitive walkthrough. These methods were deemed appropriate as this work is exploratory and little has been done in this particular context. Through the prescribed methods, we identified usability problems associated with the interpretation of an electronic fetal monitor display and associated graphical output.

\section{HCI Methods for Health Care Service}

Participatory design approaches are often used in HCI to support user-centered outcomes. Approaches that seek to involve users deeply in the design process are often lacking in the design of health

${ }^{2}$ Quote 2: http://www.babble.com/CS/blogs/strollerderby/archive/2007/10/29/fetalmonitor-inventor.aspx technologies. Kushniruk and Borycki [22] assert that HCI issues might be the most serious barriers to the successful implementation of healthcare technologies in general. Two commonly used HCI techniques, in concert with the overarching goal of this study, to garner requisite $\mathrm{HCI}$ insight are heuristic and cognitive walkthroughs.

The cognitive walkthrough method, offering a strong empirical base substantiating its efficacy in identifying usability problems, is a popular inspection-based technique [23] that focuses on ease of learning as an attribute of usability [24]. Intended especially to help understand the usability of a system for first-time or infrequent users, the cognitive walkthrough entails the researcher walking through the design in the context of representative tasks. The actions and associated feedback of the design are compared to the user's knowledge and goals [25], with discrepancies in the user's expectations and steps required by the design noted [26].

Heuristic walkthroughs were developed by leveraging the best aspects of heuristic evaluations, cognitive walkthroughs, and usability walkthroughs [27]. Heuristic walkthroughs reflect a two-part inspection process [23]. The first component is essentially task focused and entails evaluators using the system to complete a representative set of typical user tasks. In the second component of the heuristic walkthrough process evaluators complete a "free-form" evaluation of the system, typically against an established set of usability heuristics such as those offered by Nielsen [24].

Specific to health technologies, these techniques are showing promise in improving usability. For example, Edwards et al. [28] found heuristic walkthroughs beneficial in evaluating and improving the usability of commercial health records. In addition, the cognitive walkthrough technique proved successful in measures of task complexity and a means to anticipate potential user problems in the development of a telemedicine application [29].

\section{Methods}

A full understanding of an electronic fetal monitor depends on the user's ability to interpret the display, as well as the ability to interpret fetal heart rate (FHR) and contraction patterns contained within the display. The overarching goal of this study was to begin to identify those serious usability problems that could impact the patient's interpretations; and thusly, their experience. In meeting this goal, a two-part study was presented by Cockton and Woolrych, using usability inspection techniques through uncovering serious usability problems that could impact service delivery and experience. This study was exploratory and designed to uncover ideas that could be explored in larger, future studies.

In the first part of this study, heuristic walkthroughs were used to identify usability issues within the display of Philips' Avalon FM20 Antepartum and Avalon FM30 Intrapartum electronic fetal monitors that impede a layperson's ability to understand the display. In the second part of this study, cognitive walkthroughs were used to identify logic errors during interpretations of fetal heart rate graphs. In concert with the goal of this study, eight participants were recruited; this is an appropriate sample size for these methods. The participants, three males and five females, none had formal medical training. The participants were at least 18 years of age $(\mathrm{Mean}=36, \mathrm{SD}=11.5)$.

\section{Part one: Heuristic walkthrough of electronic fetal monitor display}

In the first part of the study, participants analyzed an electronic fetal 
monitor display using a heuristic walkthrough that was modeled after the two-pass process [27]. In the first pass of the heuristic walkthrough, participants were given a screen-shot printout of the display of a Philip's Avalon FM20 Antepartum and FM30 intrapartum electronic fetal monitor and were asked to label each separate designated key feature. The participant's display had lines pointing to key features on the monitor. The participant was asked to explain their thought process, using a cognitive think aloud method [30], as they wrote an appropriate label for each feature. Using the heuristic walkthrough approach, there was no set order to the tasks of labeling the display. In the second pass of the heuristic walkthrough, the participants were asked to analyze the usability of the display using heuristics defined by Nielsen [31]. Six out of the ten heuristics were chosen based on their relevance to analyzing a static screen-shot; these included visibility of system status, match between system and real world, consistency and standards, recognition rather than recall, flexibility and efficiency of use, and aesthetics and minimalist design [31].

Each of the labeled displays was compared to the diagram that Philips provided in the brochure for the Avalon FM20 Antepartum and FM30 Intrapartum. Participant responses were analyzed based on their written responses as well as their verbal responses. Responses were evaluated on the use of key terminology for display functions, which are labeled in Figure 2. Once this was determined, a percentage score for each participant was calculated from the amount of labels each participant correctly identified. The participants' thought processes were documented in a narrative form. The thought processes were analyzed to see whether the participants had any errors in their logic and compared with participants' correct or incorrect conclusions. The errors in thought processes, in conjunction with the heuristic analysis completed by the participants, were used to derive a list of usability problems in the interface with references to those usability principles that were violated by the design in each case [31]. This list was used to make suggestions for improvement to the design of the interface.

\section{Part two: Cognitive walkthrough of electronic fetal monitor graph}

In the second part of the study, participants interpreted information from an electronic fetal monitor graph set during a cognitive walkthrough [32]. Participants were asked to examine seven contractions and fetal heart rate graph sets to determine whether or not the graph sets indicated a potential threat to the baby. Graph sets previously published by Sweha et al. [33] were used during this study. Participants were shown a sequence of seven graph set printouts from an electronic fetal monitor in a set order. The participants were assigned the role of a patient or a non-medical person witnessing a mother's labor. The participants were shown the first printout and first asked to identify which graph of the graph set represented the mother's contractions and which graph represented the baby's heart rate. Before proceeding, the participants were told whether or not they identified the graphs correctly. Starting with the first graph set, the participants were then asked whether or not the printout indicated a potential complication. The participants talked through their thought process as they came to a conclusion. Participants' verbal responses were audio recorded and transcribed into a narrative form for later review and analysis. The same procedure was used for the other six printouts. At the end of the interview, participants were asked basic demographic questions.

Three aspects of participants' verbal responses were analyzed on the identification of the fetal heart rate (FHR) and contraction tracing, identification of status state, and if the participant's decision was based on appropriate design characteristics. First, the percentage score of how many participants accurately identified the FHR and contraction tracings in each graph set correctly was calculated. Second, the participants' responses for each graph set were scored to see if they accurately identified each graph set's status state as normal or not normal. Normal was defined as no complications or reason for a patient or a physician to be concerned. Not normal was defined as complications or possible complications or any reason for a patient or a physician to be concerned. For each graph set, each participant's score was calculated as a percentage and these scores were averaged for an overall score of the sample. Third, the participants' conclusions were analyzed to determine if the decision was based on appropriate design characteristics. For example, correct interpretation of an FHR tracing required the user to identify the baseline, long-term and beat-to-beat variability, and acceleration and deceleration characteristics. For the purposes of this study, correct logic was operationally defined as the

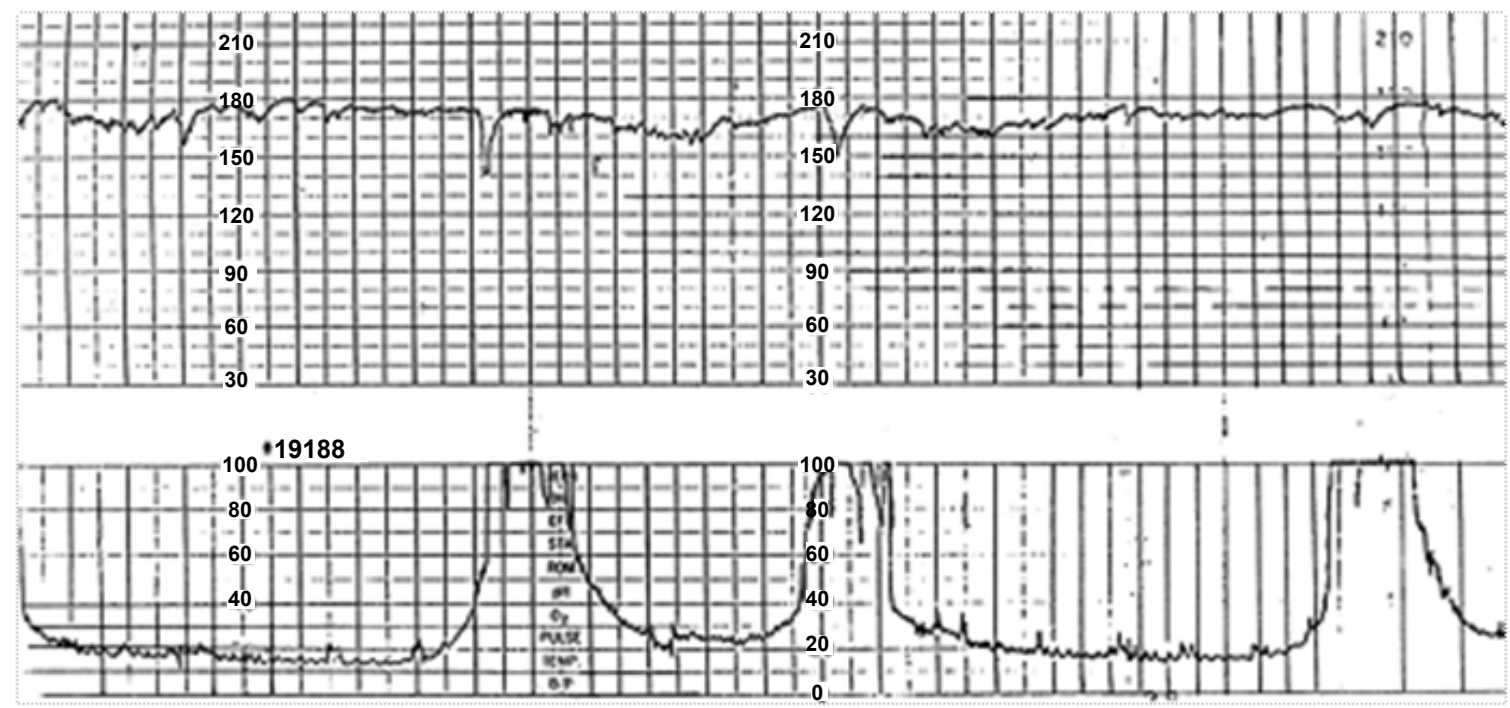

Figure 2: Abnormal FHR pattern graph used during cognitive walkthrough [33] 
correct identification of status state based on correct interpretation of appropriate design characteristics. A logic error was operationally defined as any identified status state that was not based on correct interpretation of appropriate design characteristics. The primary researcher coded each participant's response as either "correct logic" or "logic error". For example, a participants' interpretation of an FHR tracing was coded as "logic error" if the conclusion was not based on correct identification of the baseline, long-term and beat-to-beat variability, and acceleration and deceleration characteristics.

\section{Results}

During the heuristic analysis of the electronic fetal monitor display, the participants correctly identified on average $21 \%$ of the key features. Figure 3 shows the distribution of accuracy scores for all participants. Table 1 shows the heuristics that were used during the heuristic analysis.

Obtained feedback was analyzed to discern the aspects of the design that were helpful or problematic, summarized in Table 2. The table is organized in categories based on the features that the participant commented on; each comment references the heuristic that it refers to. Each entry is mapped to a particular heuristic by the number in brackets, which corresponds to the code listed in Table 1.

During the cognitive walkthrough of the electronic fetal monitor tracings, the participants correctly identified, with an average accuracy of $71 \%$, whether or not the graph set appeared normal. Figure 1 shows the distribution of accuracy scores for all the participants. Five out of eight participants identified the graphs correctly with the contraction graph on the bottom and the fetal heart rate graph on the top in the diagram.

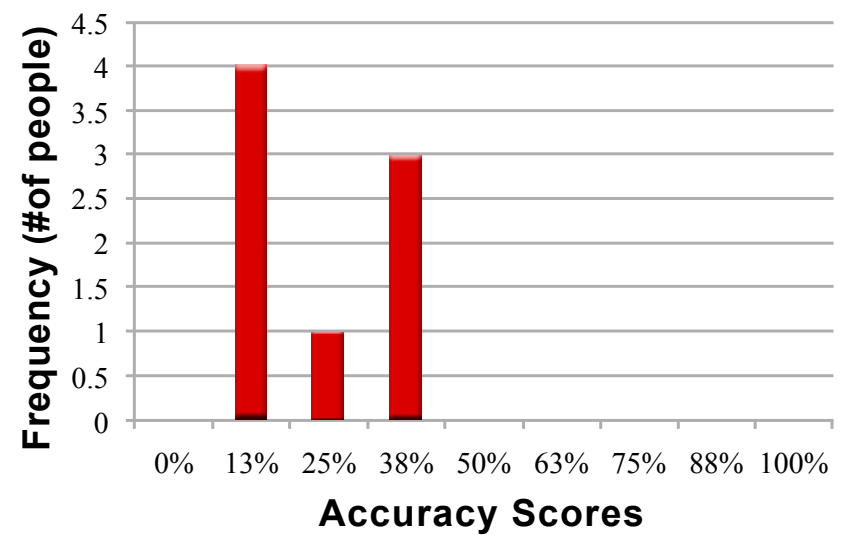

Figure 3: Distribution of Display Interpretation Accuracy Scores.

\begin{tabular}{|l|l|}
\hline Code & Heuristic \\
\hline 1 & Visibility of System Status \\
\hline 2 & Match between System and the Real World \\
\hline 3 & Consistency and Standards \\
\hline 4 & Recognition Rather than Recall \\
\hline 5 & Flexibility and Efficiency of Use \\
\hline 6 & Aesthetic and Minimalist Design \\
\hline
\end{tabular}

Table 1: Usability Heuristics Used during Heuristic Walkthrough.

\section{Discussion}

From the feedback gathered in the heuristic walkthrough analysis, there is more information than is needed being provided on the display for the patient, as conjectured. The monitor display is foreign to the patient and not usable from their perspective as a passive user. In general, one would need medical knowledge or training in order to correctly identify all of the features. This is based on the $21 \%$ accuracy score achieved by the participants overall in identifying the features. During the walkthrough the "toco" function, representing the contraction activity, was not recognized by any of the participants because they were not familiar with the terminology. One participant tried to come up with some logic for the design but became distracted by the green color, assuming it was an indicator of "good". This participant noted that the feature was in a very prominent location, which should make it an easily identifiable feature. If this feature had a label including the word "contraction", then the participants may have been able to identify the information being provided.

Two participants thought that the overall display was unattractive aesthetically because of the old, gray computer type look to it. Participants claimed that the monitor provided an easy interface for identifying such data as blood pressure, contractions, and heartbeat. However, based on participant performance during the walkthrough, participants did not identify these features correctly the majority of the time.

The black background and large, colored numbers provide good contrast that makes the monitor easy to read. Users seemed to appreciate the consistent, aesthetically pleasing color-coding for features that related to one another. However, when analyzing participants' abilities to identify these same features, most saw a relationship between FHR1 and FHR2, but none noticed the color-coding between the mother's heart rate and the cardiogram. Therefore, none were able to label the mother's cardiogram correctly. This mistake indicates that the majority of the participants focused solely on the task at hand and did not view the entire spectrum of context clues that were provided.

Participants were not able to discern that both heart rates, FHR1 and FHR2, were for babies. Out of the participants that were able to correctly identify FHR1, only one had correct logic to support this decision. One participant knew that a baby's heart rate is higher than an adult's heart rate but assumed that FHR2 was the mother's heart rate, thus making FHR1 the baby's heart rate. Another participant claimed that the electronic fetal monitor does not display the mother's heart rate, therefore assuming that FHR1 must be for the baby. However, on this model, the mother's heart rate was shown as well. There was also a total of three guesses, or non-supported responses, which brought participants to the correct conclusion. One participant that incorrectly labeled FHR1 and FHR2 determined that an adult's heart rate is lower than a baby's heart rate, but this led the participant to label FHR1 as the mother's heart rate. In this case though, the heart rate for the baby just happened to be abnormally low at the time of the screen shot. This mistake was also made when it came to identifying FHR2 because this logic was carried through.

The symbol representing the auditory feature provided a standard icon that three of the participants easily identified during the walkthrough. Most participants were able to identify the warning symbol. But as it fits into the visibility on the monitor, the warning symbol should be larger due to its importance in the context. One 


\begin{tabular}{|c|c|c|}
\hline Design Aspect & Good & Problematic \\
\hline System as a whole & $\begin{array}{l}\text { - Easily identify key information: contraction, blood } \\
\text { pressure, heart beat [1] } \\
\text { - Straight forward, easy to use [5] } \\
\text { - Usable for experienced user (maybe inexperienced as well) } \\
\text { [5] } \\
\text { - Gives vital information } \\
\text { - Ugly [6] }\end{array}$ & $\begin{array}{l}\text { - Too much information for a patient [1] } \\
\text { - Very data driven and "techie" [2] } \\
\text { - Foreign technology to a patient [2] } \\
\text { - Not able to definitely identify features - guessing necessary [4] } \\
\text { - Experimentation with device needed for understanding [5] } \\
\text { - Not flexible to new user [5] } \\
\text { - Reduce number of features [5] }\end{array}$ \\
\hline Layout & $\begin{array}{l}\text { - Clean, not crowded for easy readability [1] } \\
\text { - Aesthetically pleasing [6] }\end{array}$ & $\begin{array}{l}\text { - Need medical knowledge to identify all features } \\
\text { - Layout not consistent for every feature [3] }\end{array}$ \\
\hline Background & - Black background provides good contrast [1] & - Red on black is not easy to read [6] \\
\hline $\begin{array}{l}\text { Fetal Heart Rate } \\
\text { Numbers }\end{array}$ & $\begin{array}{l}\text { - Large, spread out, colored for easy readability, good } \\
\text { contrast, sharp [1] } \\
\text { - Color coding (ex. Yellow= warning) [1] } \\
\text { - Consistent colors used [3] } \\
\text { - Font larger for more important numbers consistently [3] } \\
\text { - FHR symbols look the same and have same meaning [3] } \\
\text { - Based on heart rate, contraction can be identified when rate } \\
\text { goes up or down [4] } \\
\text { - Color aesthetically pleasing [6] }\end{array}$ & - Small numbers difficult to read [1] \\
\hline Symbols/Pictures & $\begin{array}{l}\text { - Pictorial cardiograph good for quick viewing [1] } \\
\text { - Cardiograph big clue of healthcare [4] }\end{array}$ & - Lack of pictorial usage for conducive for patient [2] \\
\hline Warning & $\begin{array}{l}\text { - Smaller size warning may startle patient less [1] } \\
\text { - Auditory feature to alert of a problem [1] }\end{array}$ & $\begin{array}{l}\text { - Not obvious what it relates to and should be larger since it is an } \\
\text { important warning [1] }\end{array}$ \\
\hline Buttons & $\begin{array}{l}\text { - Buttons all consistently the same size and format [3] } \\
\text { - Appear as touch screen [4] }\end{array}$ & $\begin{array}{l}\text { - Menu button lacking graphics and not consistent with computer standards } \\
\text { such as Windows [3] }\end{array}$ \\
\hline
\end{tabular}

*Bold comments represent the feedback that was mentioned more than once

Table 2.Design Pros and Cons Identified during Heuristic Analysis.

participant, however, questioned whether smaller would be better to reduce panic for the patient. The buttons across the bottom of the monitor are consistent and have a touch screen look to them. However, a participant suggested adding graphics to make the meaning of each button more clear. When it came to participants actually identifying some of the purposes of the buttons, the majority could not do so easily. Although the forward button was very familiar to most participants, the difficulty in identifying this feature accurately was that no one could figure out what extra information would be shown by using this option.

From this feedback, a possible way of providing the patient with less information, while also preserving the information that is needed for healthcare professionals, would be possibly use a separate screen or display that can be customized to show patient information that is tailored to patient needs. Based on the limited amount sample in this study, a more extensive study should be performed to develop additional design recommendation for this stakeholder group.

When interpreting the information provided by the electronic fetal monitor graph sets, it is evident that just coming to a correction conclusion does not mean that the participant had correct logic when coming to that conclusion. These results indicate that if patients are not provided with basic information, even just which graph represents the contraction and which graph represents the fetal heart rate, the patients might misinterpret the feedback from the graph. "If we are fortunate, thoughtful designers provide the clues for us. Otherwise, we must use our creativity and imagination". Organizationally, patients should be informed by their physicians and nurses what to look for in the graph, if nothing else just to comfort the patient. However, given the limited resources and varied nature of patients (e.g. education, urgency, health status, prior experiences etc.) and organizations (e.g. appropriate staffing, time pressures, etc.) this bedside education about the technology may not be possible.

\section{Conclusion}

This study shows that there are several issues related to patients being able to interpret information on an electronic fetal monitor display. When patients cannot understand what technologies are saying about their health status and bodily functions they are forced to make decisions based on incomplete or inaccurate information. The results of this type of biased decision-making can have negative impacts of the provision of health service, patient health outcomes, and patient care seeking behaviors.

A lack of knowledge, on the part of the patients, family, and friends can inhibit accurate interpretation of the graphs. If efforts are made to educate passive users, and improve the design itself, patients and others will be able to better interpret and be comforted by the information on an electronic fetal monitor, enhancing the overall care provision experience. To ensure a universal design, which can be interpreted by all potential users of the electronic fetal monitor, including medical practitioner, patients, and family members, designers should particularly avoid medical jargon in display labels, use consistent, aesthetically pleasing color-coding for features and consider customized screens or presentations of information for different user populations. Future research in this area should explore the effects of patients positive and negative experiences with technologies used in their care on system and quality outcomes. Little is known about the effects of patient attitudes about technologies on their ratings of quality. There is also a need to understand how patient attitudes affect variables such as interruptions. Interruptions have been found to lead to errors [34,35]. When patients do not understand displays or incorrectly interpret displays they may interrupt providers and other care processes. Future research should also explore passive users in monitoring technologies in other health services sectors such as intensive care units. 
Citation: Brickel S, Montague E, Winchester WW (2012) Patients User Experiences with Technologies in Health Service Industry. J Health Med Informat S7:002. doi:10.4172/2157-7420.S7-002

\section{Acknowledgements}

This publication was supported by grant 1 UL1RR025011 from the Clinical \& Translational Science Award (CTSA) program of the National Center for Research Resources National Institutes of Health and award number 0851774 from the National Science Foundation in support of REU Site: Building Interfaces for Tomorrow's Technology -The Virginia Tech Research in Human-Computer Interaction Program. The University of Wisconsin-Madison Systems Engineering Initiative for Patient Safety (SEIPS) provided support on this project http://cqpi. engr.wisc.edu. The authors would also like to thank Kim Gausepohl and Scott McCrickard for their feedback on earlier versions of this manuscript.

\section{References}

1. Montague E (2009) Patient user experience of medical technology. In Karsh BT (Edn), Ergonomics and Health Aspects of Work with Computers, International Conference, EHAWC 2009, Held as Part of HCI International 2009 (pp. 70-77). San Diego, CA, USA.

2. Nerum H, Halvorsen L, Sørlie T, Oian P (2006) Maternal request for cesarean section due to fear of birth: can it be changed through crisis-oriented counseling? Birth 33: 221-228.

3. Thom DH, Kravitz RL, Bell RA, Krupat E, Azari R (2002) Patient trust in physician: relationship to patient requests. Fam Pract 19: 476-483.

4. Young D (2006) "Cesarean Delivery on Maternal Request": was the NIH conference based on a faulty premise? Birth 33: 171-174.

5. Inbar O, Tractinsky N (2009) The incidental user. Interactions 16: 56-59.

6. Tien JM, Goldschmidt-Clermont PJ (2009) Engineering Healthcare As A Service System. Stud Health Technol Inform 153: 277-97.

7. Berry LL, Bendapudi N (2007) Healthcare: A Fertile Field for Service Research. Journal of Service Research 10: 111-122.

8. Kleiner BM (2007) Sociotechnical system design in health care. In P. Carayon (Edn), Handbook of Human Factors and Ergonomics in Health Care and Patient Safety. Mahwah: Lawrence Erlbaum Associates.

9. Coiera E (2004) Four rules for the reinvention of health care. BMJ 328: 11971199.

10. Carayon P (2006) Human factors of complex sociotechnical systems. App Ergon 37: 525-535.

11. Stephen Timmons, Russell Harrison-Paul, Brian Crosbie (2008) How do lay people come to trust the Automatic External Defibrillator? Health Risk Soc 10: 207-220.

12. Montague EN, Winchester WW, Kleiner BM (2010) Trust in Medical Technology by Patients and Health Care Providers in Obstetric Work Systems. Behav Inf Technol 29: 541-554

13. Bate P, Robert G, Lynne M (2007) Bringing User Experience to Healthcare Improvement: The Concepts, Methods and Practices of Experience-based Design. (1stedn), Oxford: Radclife Publishing.

14. Martin JA, Hamilton BE, Sutton PD, Ventura SJ, Menacker F, et al. (2006) National Vital Statistics Reports: Births: US Department of Health and Human Services.

15. Yang YT, Mello MM, Subramanian SV, Studdert DM (2009) Relationship between malpractice litigation pressure and rates of cesarean section and vaginal birth after cesarean section. Med Care 47: 234-242.

16. Carter MC (1988) Fetal monitoring. J Biomed Eng 10: 527-532.

17. NIH (2009) Fetal heart monitoring. Retrieved July 17.

18. Feinbloom RI (1993) Pregnancy, birth and the early months (2ndedn), Reading Massachusetts: Addison-Wesley Publishing Company

19. Martin EJ (2002) Intrapartum management modules: a perinatal eduation program (3rdedn), Philadelphia: Lippincott Williams and Wilkins.
20. Boos A, Jagger MH, Paret GW, Hausmann JW (1995) A new, lightweight feta telemetry system. Hewlett-Packard Journal 82-93.

21. Philips (2006) Avalon FM20 Antepartum and FM30 Intrapartum.

22. Kushniruk A, Borycki E (2007) Human factors and usability of healthcare systems. In Bardram $\mathrm{J}$ and Mihailidis $\mathrm{A}$ (Edn) Pervasive computing in healthcare. New York: CRC Press.

23. Sears A (1997) Heuristic Walkthroughs: Finding the Problems Without the Noise. Int J Hum Comput Interact 9: 213-234.

24. Nielsen J (1994) Usability inspection methods. New York: Wiley.

25. Connell I, Blandford A, Green T (2004) CASSM and cognitive walkthrough: usability issues with ticket vending machines. Behav Inf Technol 23: 307-320.

26. Jeffries R, Miller JR, Wharton C, Uyeda K (1991) User interface evaluation in the real world: A comparison of four techniques. Paper presented at the Proceedings of CHI'91, New Orleans, LA.

27. Sears AL (1995) Heuristic walkthroughs: Finding problems without the noise. Int J Hum Comput Interact 9: 213-234.

28. Edwards PJ, Moloney KP, Jacko JA, Sainfort F (2008) Evaluating usability of a commercial electronic health record: A case study. Int J Hum Comput Interact 66: 718-728.

29. Kaufman DR, Patel VL, Hilliman C, Morin PC, Pevzner J, et al. (2003) Usability in the real world: assessing medical information technologies in patients homes. J Biomed Inform 36: 45-60.

30. Ericsson KA, Simon HA (1993) Protocol Analysis: Verbal Reports as Data. Cambridge, MA: MIT Press.

31. Nielsen J (2005) Ten usability heuristics. Retrieved July 62007.

32. Polson PG, Lewis C, Rieman J, Wharton C (1992) Cognitive walkthroughs: a method for theory-based evaluation of user interfaces. Int J Man Mach Stud 36: 741-773.

33. Sweha A, Hacker TW, Nuovo J (1999) Interpretation of the electronic fetal heart rate during labor. Am Fam Physician 59: 2487-2500.

34. Chisholm CD, Collison EK, Nelson DR, Cordell WH (2008) Emergency department workplace interruptions: are emergency physicians "interruptdriven" and "multitasking". Acad Emerg Med 7: 1239-1243.

35. Flynn EA, Barker KN, Gibson JT, Pearson RE, Berger BA, et al. (1999) Impact of interruptions and distractions on dispensing errors in an ambulatory care pharmacy. Am J Health Syst Pharm 56: 1319-1325. 